06

\title{
Численное моделирование поведения карбидов при высокоэнергетическом воздействии
}

\author{
(C) К.К. Маевский ${ }^{1,2}$ \\ ${ }^{1}$ Институт гидродинамики им. М.А. Лаврентьева СО РАН, \\ 630090 Новосибирск, Россия \\ ${ }^{2}$ Новосибирский государственный университет, \\ 630090 Новосибирск, Россия \\ e-mail: konstantinm@hydro.nsc.ru
}

Поступило в Редакцию 1 июля 2021 г.

В окончательной редакции 13 сентября 2021 г.

Принято к публикации 17 сентября 2021 г.

Представлены результаты моделирования термодинамических параметров ударно-волнового нагружения карбидов с различными стехиометрическими соотношениями. Выполнены расчеты значений давления, сжатия и температуры для сплошных и пористых карбидов в диапазоне значений давления выше $3 \mathrm{GPa}$. Проведено сравнение модельных расчетов с известными экспериментальными результатами по ударноволновому нагружению карбидов различных значений пористости. Показана возможность моделирования по предложенной методике поведения для карбидов, по которым отсутствуют экспериментальные данные, при высоких динамических нагрузках.

Ключевые слова: уравнение состояния, ударная адиабата, термодинамическое равенство, пористая гетерогенная среда, карбиды.

DOI: $10.21883 / J T F .2022 .01 .51858 .200-21$

\section{Введение}

Для решения большого количества задач в различных высокотехнологичных отраслях промышленности наиболее эффективны высокотемпературные композиционные материалы. В настоящее время интенсивно изучаются образцы на основе безоксидных тугоплавких соединений-боридов, карбидов, нитридов. Опираясь на принципы и возможности порошковой, в том числе керамической технологии, можно создать с помощью этих тугоплавких соединений многочисленные композиционные материалы для различных практических применений [1]. Исследованию карбидов и материалов с ними в качестве компонентов посвящено много работ, например [2-26]. Одно из направлений исследований сжимаемости карбидов связано с проблемами взрывного компактирования порошков карбидов, получения микрообразцов этих соединений, обладающих твердостью исходных крупинок образцов [2].

Повышенный интерес среди карбидов, в частности, для производства керамик и композитов вызывает карбид бора $\mathrm{B}_{4} \mathrm{C}$, уступающий по значению твердости только алмазу [3-17]. Несмотря на то что исследования по ударному сжатию $\mathrm{B}_{4} \mathrm{C}$ продолжаются уже длительное время, начиная с первых статей [3-5], вопрос о наличии фазового перехода в диапазоне давлений от 10 до $100 \mathrm{GPa}$ остается предметом дискуссий. Предположение о возможности фазового перехода в карбиде бора при ударном сжатии были высказаны в [6]. В работах $[7,8]$ сделаны предположения о фазовых превращениях карбида бора при ударном сжатии на основании наличия изломов ударной адиабаты. При этом, хотя в [7], как и в предыдущих исследованиях, есть предположения об одном или нескольких фазовых переходах, там отмечается, что данные эксперименты не дают четких доказательств переходов, экспериментальные данные согласуются с фазовым переходом с небольшим изменением объема при значении давления около $40 \mathrm{GPa}$. В [8] высказано предположение, что отличие $\mathrm{B}_{4} \mathrm{C}$ от других материалов заключается в том, что ступенчатая структура не будет появляться в профиле ударной волны. Изменение структуры или механизм этого фазового перехода могут отличаться от других хрупких материалов благодаря своей уникальной кристаллической структуре. Для более четкого понимания перехода необходимы дальнейшие исследования при статическом и ударном сжатии [8]. В обзорной работе [9] описаны представления об ударно-волновом нагружении карбида бора в диапазоне давлений до $90 \mathrm{GPa}$. При этом оказалось, что точно определить давление фазового превращения ударно-сжатого карбида бора затруднительно в связи с сильным разбросом экспериментальных данных, обусловленным различием в стехиометрии, пористости и способе изготовления образцов. Выводы, сделанные в [9] по поводу возможности фазового перехода, как отмечают авторы, спорны, но представляют собой разумную оценку имеющейся ударной волны и подтверждающих данных. Отмечается, что $\mathrm{B}_{4} \mathrm{C}$ является и, вероятно, останется одним из наиболее загадочных материалов среди керамик, исследуемых ударно-волновыми методами [9]. Факт фазового превращения со скачкообразным изменением объема при ударном сжатии кристаллического 
$\mathrm{B}_{4} \mathrm{C}$ не находил подтверждения в экспериментах по статическому сжатию [10], где уравнение состояния и механизм сжатия почти стехиометрического $\mathrm{B}_{4} \mathrm{C}$ исследованы до максимального квазигидростатического давления $74 \mathrm{GPa}$. Объемное сжатие $\mathrm{B}_{4} \mathrm{C}$ в экспериментах не демонстрирует фазовых переходов вплоть до $70 \mathrm{GPa}[11]$. В то же время в [12] отмечается, что совокупность всех экспериментальных данных показывает плато при значении волновой скорости $12.5 \mathrm{~km} / \mathrm{s}$, что может свидетельствовать о наличии фазы высокого давления. В [13] выявлен излом ударной адиабаты $\mathrm{B}_{4} \mathrm{C}$ при давлении $100 \mathrm{GPa}$, который соотнесен с началом плавления $\mathrm{B}_{4} \mathrm{C}$ при ударном сжатии.

Кроме высокой твердости, карбиды характеризуются высокой температурой плавления. Основой для производства высокотемпературных материалов являются тугоплавкие карбиды, обладающие большими значениями твердости и устойчивостью к коррозии. В ядерной технике защитные оболочки микротвэлов высокотемпературного реактора изготавливаются из слоев карбидов и графита, предназначенных для длительной работы при высоких температурах [18]. При этом понимание свойств уран-углеродных (UC) систем важно не только в связи с их потенциальной кандидатурой для применения в атомной промышленности, но и с точки зрения фундаментальной науки. Однако что касается поведения UC при высоком давлении, в литературе существует очень мало экспериментальных и теоретических исследований по этому материалу [19-23]. Похожая ситуация наблюдается и с другими тугоплавкими карбидами, в частности с карбидами тантала ТaC и гафния HfC, а также с их смесями, которые имеют аномально высокие температуры плавления около $4000 \mathrm{~K}$, и при этом сохраняют высокую прочность и долговечность при экстремальных тепловых нагрузках. В работе [24] отмечается, что на сегодняшний день в литературе практически отсутствуют данные о свойствах этих карбидов при высоких температурах, возникающих в аварийных режимах работы оборудования, что затрудняет моделирование и управление такими режимами.

Как отмечается в [25], „высокотвердые керамики... часто используются в условиях, сопряженных с интенсивными ударными воздействиями. В силу дороговизны сложных керамических изделий актуальным становится прогнозирование результатов ударных воздействий методами компьютерного моделирования“. Один из возможных вариантов моделирования поведения карбидов показан в [26], где карбиды рассматривались как смесь компонентов, входящих в состав исследуемого образца. Смесевая модель, при которой предполагается, что компоненты сжимаются в смеси так же, как и чистые материалы, позволила достоверно описывать данные ударно-волновых экспериментов сплошных карбидов с равным стехиометрическим соотношением компонентов $\mathrm{WC}, \mathrm{TiC}, \mathrm{TaC}, \mathrm{NbC}, \mathrm{SiC}, \mathrm{ZrC}$. Карбид титана ТiC интересен тем, что является тугоплавким, сверхтвердым, износостойким материалом с высокой электро- проводностью и стойкостью к коррозии [27-30]. Также наряду с высокопрочными и высокоплотными сплавами на основе вольфрама, которые обладают высокой проникающей способностью при ударном взаимодействии, определенный интерес представляют собой пористые композиционные материалы на этой же основе, легированные высокопрочными компонентами типа карбидов вольфрама [31,32].

C учетом необходимости определения параметров карбидов, имеющих различные значения пористости, при высоких динамических нагрузках, а также для карбидов, по которым мало или отсутствуют экспериментальные данные, желательно иметь достаточно простую модель, позволяющую получать достоверные оценки термодинамических характеристик нагружаемых сложных материалов. В частности, для описания пористых материалов необходимо учитывать взаимодействие компонентов, что не предполагается при использовании смесевого метода. В [33-37] была рассмотрена термодинамически равновесная модель ТЕС (thermodynamic equilibrium components) применительно к ряду материалов, в том числе и к карбидам [14].

Целью настоящей работы является моделирование ударно-волнового нагружения карбидов с различными значениями пористости, при этом исследуемый материал рассматривается как смесь составляющих его компонентов. Используемый подход позволяет не только описывать данные, полученные на основании эксперимента для карбидов с различными стехиометрическими соотношениями компонентов, но и определять термодинамические характеристики ударно-волнового нагружения ранее не исследованных карбидов.

\section{1. Методика расчета}

Для описания ударно-волнового нагружения карбидов, рассматриваемых как смесь компонентов, используется модель ТЕС. Данная модель основана на предположении, что все компоненты смеси, включая газ в порах, находятся в термодинамическом равновесии, которое предполагает равенство скоростей, давлений и температур. В рассматриваемой модели для описания поведения конденсированных фаз используется уравнение состояния типа Ми-Грюнайзена. Выписываются уравнения, определяющие состояние конденсированного компонента:

$$
\begin{gathered}
P(\rho, T)=P_{C}(\rho)+P_{T}(T), \\
P_{T}(\rho, T)=\Gamma \rho E_{T}(T), \quad E_{T}(T)=c_{V}\left(T-T_{0}\right),
\end{gathered}
$$

где $P_{C}-$ потенциальная компонента давления, $P_{T}, E_{T}$ - тепловые компоненты давления и удельной энергии, $c_{V}$ - теплоемкость, $T_{0}-$ начальная температура. Тогда уравнение состояния для конденсированного компонента с текущей и начальной плотностью $\rho, \rho_{0}$ и 
Параметры модели ТЕС

\begin{tabular}{c|c|c|c|c|c|c|c|c}
\hline Материал & $A, \mathrm{GPa}$ & $\rho, \mathrm{g} / \mathrm{cm}^{3}$ & $k$ & $c_{v}, \mathrm{~J} /(\mathrm{kg} \cdot \mathrm{K})$ & $\Gamma\left(T_{0}\right)$ & $\Gamma\left(T_{*}\right)$ & $T_{*}, \mathrm{~K} \cdot 10^{3}$ & $\Gamma\left(T_{\infty}\right)$ \\
\hline $\mathrm{C}$ & 200.0 & 3.515 & 2.6 & 500 & 1.10 & 0.55 & 20 & 0.500 \\
\hline $\mathrm{W}$ & 101.8 & 19.235 & 3.105 & 140 & 1.61 & 1.35 & 23 & 0.0 \\
\hline $\mathrm{Nb}$ & 52.0 & 8.586 & 3.2 & 280 & 1.68 & 1.20 & 23 & 0.500 \\
\hline $\mathrm{B}$ & 115.0 & 2.340 & 1.7 & 816 & 1.10 & 0.90 & 23 & 0.500 \\
\hline $\mathrm{Ta}$ & 54.18 & 16.656 & 3.45 & 140 & 1.69 & 1.15 & 23 & 0.667 \\
\hline $\mathrm{U}$ & 35.0 & 19.040 & 4.0 & 180 & 1.83 & 1.20 & 23 & 0.500 \\
\hline $\mathrm{Mo}$ & 95.0 & 10.206 & 3.0 & 294 & 1.58 & 1.15 & 23 & 0.500
\end{tabular}

соответствующими коэффициентами $A, k$ имеет вид

$$
P(\rho, T)=A\left(\left(\frac{\rho}{\rho_{0}}\right)^{k}-1\right)+\Gamma c_{V}\left(T-T_{0}\right) \rho .
$$

Значение давления и внутренней энергии в нормальных условиях $\left(\rho=\rho_{0}, T=T_{0}\right)$ в рассматриваемой модели полагаются равными нулю. Для описания поведения материалов различной пористости в уравнение состояния вводится функция $\Gamma=P_{T} V / E_{T}$, определяющая вклад тепловых компонент и зависящая от температуры, в следующем виде:

$$
\begin{gathered}
\Gamma(T)=\left[\left(\Gamma\left(T_{0}\right)-\Gamma\left(T_{\infty}\right)\right)^{-1}+C\left(T-T_{0}\right)\right]^{-1}+\Gamma\left(T_{\infty}\right), \\
C=\left(\left[\Gamma\left(T_{*}\right)-\Gamma\left(T_{\infty}\right)\right]^{-1}-\left[\Gamma\left(T_{0}\right)-\Gamma\left(T_{\infty}\right)\right]^{-1}\right)\left(T_{*}-T_{0}\right)^{-1} .
\end{gathered}
$$

Параметры $\Gamma\left(T_{0}\right), \Gamma\left(T_{*}\right), \Gamma\left(T_{\infty}\right)$ выбираются из условия соответствия ударной адиабаты известным экспериментальным результатам исследуемых образцов:

$$
\begin{gathered}
\Gamma(T) \rightarrow \Gamma\left(T_{0}\right) \quad \text { при } \quad T \rightarrow T_{0}, \\
\Gamma(T) \rightarrow \Gamma\left(T_{\infty}\right) \quad \text { при } \quad T \rightarrow T_{\infty} .
\end{gathered}
$$

$\Gamma\left(T_{0}\right)$ берется на основании известных данных при нормальных условиях. Значение $\Gamma\left(T_{*}\right)$ соответствует температуре $T=T_{*}$, асимптотическое значение $\Gamma\left(T_{\infty}\right)$ соответствует значению температуры при максимальном сжатии $T=T_{\infty}$.

Для газа берется уравнение состояния идеального газа. На фронте волны выписываются условия сохранения массы, импульса и энергии [38], известные как законы Гюгонио. Условия сохранения импульса и энергии рассматриваются для смеси в целом, а для сохранения потока массы выписываются условия для каждого компонента смеси. Такой подход позволяет моделировать не только сжатие материала как целого, но и сжатие каждого компонента в отдельности. Для смеси, в состав которой входят $n$ конденсированных компонентов, имеющих начальные объемные доли $\mu_{n 0}$, получено следующее выражение:

$$
\begin{gathered}
P=\frac{\sum_{i=1}^{n} A_{i} \frac{\mu_{i 0}}{\sigma_{i}}\left[\left(h_{i}-\frac{k_{i}+1}{k_{i}-1}\right) \sigma_{i}^{k_{i}}+\frac{2 k_{i} \sigma_{i}}{k_{i}-1}-h_{i}-1\right]}{\sum_{i=1}^{n} \frac{\mu_{i 0}}{\sigma_{i}} h_{i}+\left(\frac{h_{g}}{\sigma_{g}}\right)\left(1-\sum_{i=1}^{n} \mu_{i 0}\right)-1}, \\
h_{i}=\frac{2}{\Gamma_{i}}+1, \quad h_{g}=\frac{2}{\gamma-1}+1 .
\end{gathered}
$$

Здесь $\sigma_{i}=\rho_{i} / \rho_{i 0}, \sigma_{g}=\sigma_{g} / \sigma_{g 0}-$ степени сжатия соответствующего компонента $i=1 \ldots n$, а $\rho_{g}, \rho_{g 0}$ - текущая и начальная плотности газа, $\gamma=1.41$ - показатель адиабаты. Полученного уравнения в совокупности с условием равенства значений температур компонентов и уравнениями состояния каждого компонента достаточно для нахождения зависимости, которую можно рассматривать как ударную адиабату многокомпонентной смеси. Для расчета поведения сплошного материала полагаем объемную долю газа равной нулю. Чистое вещество рассматривается как смесь с одним конденсированным компонентом. Это дает возможность определить параметры для компонентов исследуемых материалов. Полученные таким образом уравнения состояния чистых материалов позволяют достоверно описывать данные, полученные на основании ударно-волновых экспериментов.

\section{2. Результаты моделирования}

Наличие данных, полученных на основании экспериментов, позволило верифицировать рассматриваемую модель и рассмотреть возможности использования последней для материалов, по которым недостаточно данных или они отсутствуют. При описании ударноволнового нагружения карбидов в расчетах использовались адиабаты компонентов. Для углерода бралась адиабата алмаза аналогично [26]. Параметры модели, полученные на основе экспериментальных данных для компонентов исследуемых карбидов, показаны в таблице.

Результаты численного моделирования и экспериментальные данные для карбида вольфрама 
WC $\rho_{0}=15.66 \mathrm{~g} / \mathrm{cm}^{3}$ и для карбида ниобия $\mathrm{NbC}$ $\rho_{0}=7.8 \mathrm{~g} / \mathrm{cm}^{3}$ показаны на рис. 1,2 в переменных давление-сжатие. Сжатие $\sigma$ определяется как отношение начальной плотности образца к текущему значению плотности. Здесь же показаны ударные адиабаты и экспериментальные данные для компонентов карбидов. Значения объемных долей компонентов, используемые в рассматриваемой модели, для карбидов определялись исходя из стехиометрического соотношения элементов. Результаты моделирования достоверно описывают данные, полученные на основании экспериментов, и соответствуют расчетам, которые проводились ранее для сплошных карбидов [26] с равными долями таких компонентов.

Авторский подход позволил описать данные экспериментов не только для сплошных карбидов аналогично [26], но и для пористых образцов. Пористый карбид рассматривается как пористая смесь с компонентами

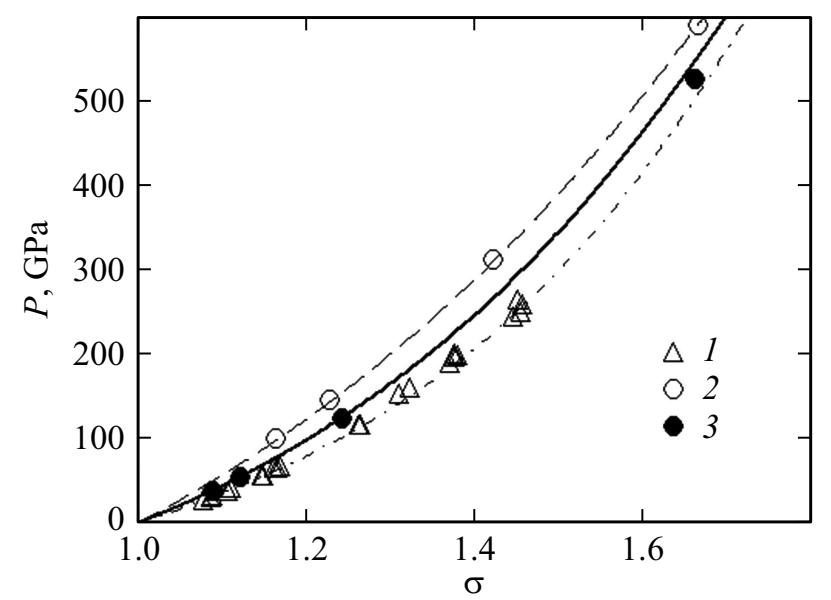

Рис. 1. Ударные адиабаты: штриховая линия для алмаза, штрихпунктирная линия для вольфрама, сплошная линия для карбида вольфрама WC. Экспериментальные данные: $1,3-[39] ; 2-[40]$.

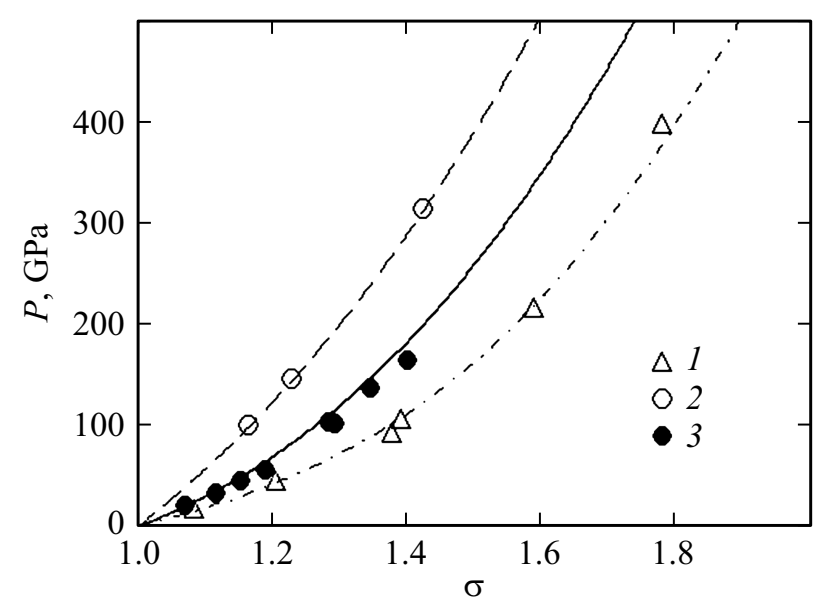

Рис. 2. Ударные адиабаты: штриховая линия для алмаза, штрихпунктирная линия для ниобия, сплошная линия для карбида ниобия NbC. Экспериментальные данные: 1 - [39], $2-[40], 3-[2]$.

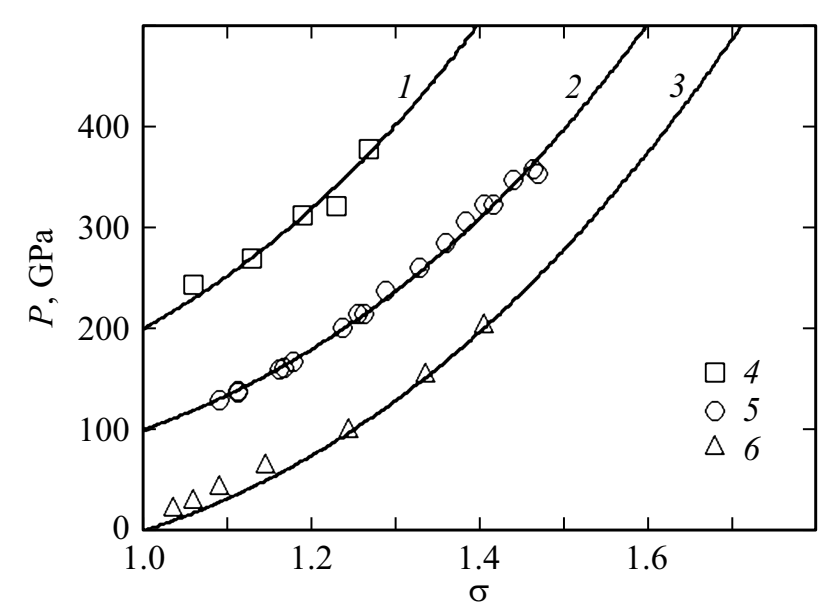

Рис. 3. Ударные адиабаты пористых карбидов. Расчет для WC $(m=1.201)-1, \mathrm{TaC}(m=1.125)-2, \mathrm{NbC}(m=1.07)-3$. Экспериментальные данные: $4-[42], 5-[41], 6-[2]$.

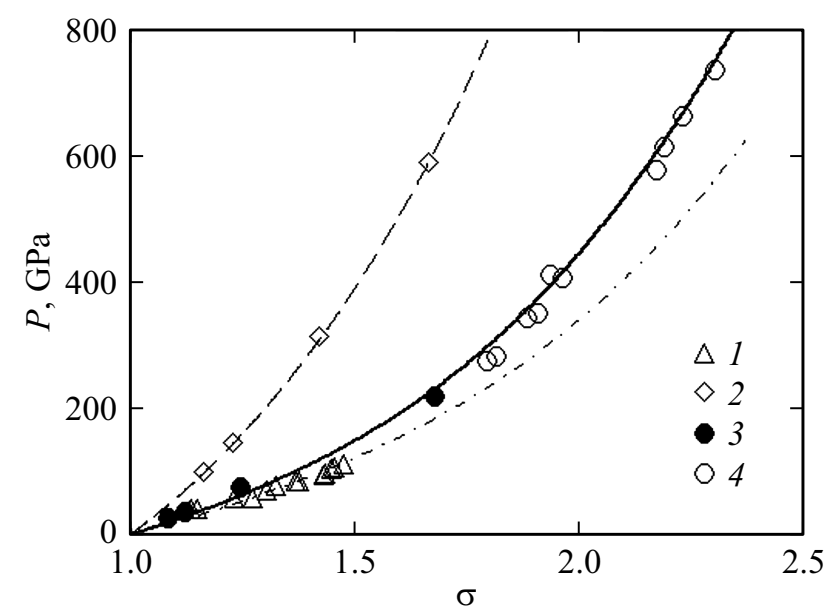

Рис. 4. Ударные адиабаты: штриховая линия для алмаза, штрихпунктирная линия для бора, сплошная линия для карбида бора $\mathrm{B}_{4}$ С. Экспериментальные данные: $1-[41] ; 2-[40]$, $3-[3], 4-[12]$.

соответствующих элементов карбида, при этом соотношение объемных долей конденсированных компонентов уменьшается пропорционально пористости образца $m$ (пористость $m$ - отношение плотности твердого вещества к плотности образца). Результаты моделирования для карбидов различных значений пористости показаны на рис. 3. Для карбида вольфрама, имеющего значение пористости $m=1.201$, карбида тантала $m=1.125$ и карбида ниобия $m=1.07$ получено описание поведения пористых материалов в пределах точности эксперимента. Для наглядности расчеты и данные показаны со сдвигом по значению давления на $100 \mathrm{GPa}$.

Предположение о возможности определения объема компонентов, основанного на стехиометрическом соотношении, позволило достоверно описать, как это показано на рис. 4, данные экспериментов и для карбида 


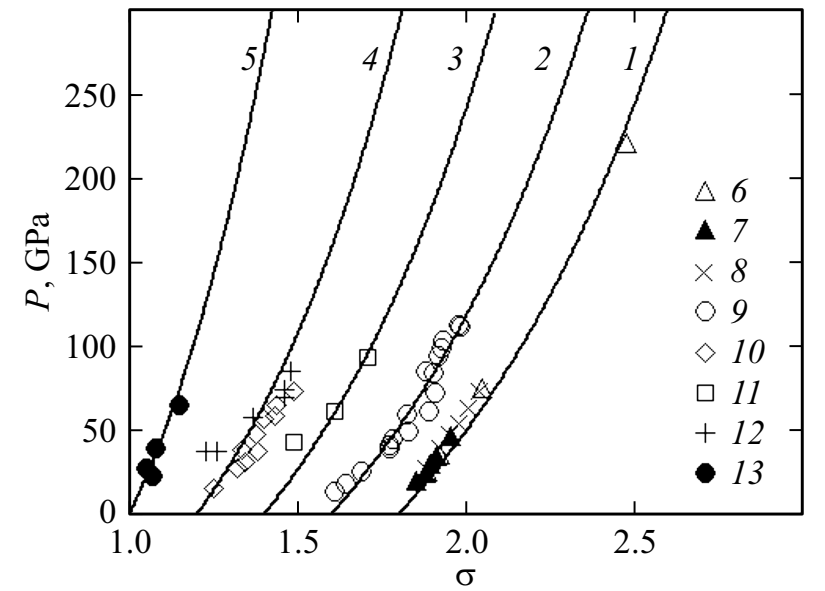

Рис. 5. Ударные адиабаты пористого карбида бора $\mathrm{B}_{4}$ С. Расчет для пористости $m=1-1, m=1.046-2, m=1.16-3$, $m=1.29-4, m=1.74-5$; Экспериментальные данные: $6-[3] ; 7,8-[7] ; 9,10-[41] ; 11-13-[42]$.

бора $\mathrm{B}_{4} \mathrm{C}$. Для $\mathrm{B}_{4} \mathrm{C}$ объемные доли определялись в соотношении 4:1 соответственно химическому составу.

Подобный подход позволяет достоверно описывать экспериментальные данные при интенсивных динамических нагрузках как для сплошных, так и для пористых образцов $\mathrm{B}_{4} \mathrm{C}$. Расчеты и соответствующие экспериментальные данные $[3,7,41]$ и данные Дудоладова из [42] для образцов карбида бора с различными значениями пористости приведены на рис. 5 (для сплошного образца $m=1)$. На основании проведенного моделирования можно сделать вывод, что в рассмотренном диапазоне значений давления, который интересен с точки зрения исследований, связанных ударно-волновым синтезом, получено удовлетворительное соответствие расчетных кривых экспериментальным данным. Отклонение расчетных кривых от данных экспериментов при значениях пористости 1.16 и 1.29 может быть связано с тем, что с ростом пористости точность определения экспериментальных данных снижается.

Полученные результаты позволяют предположить, что возможно моделировать по такой же методике термодинамические параметры карбидов с различными стехиометрическими соотношениями, для которых отсутствуют экспериментальные данные. Подобные расчеты термодинамических характеристик были проведены для карбида урана UC с равными долями таких компонентов. Объемные доли определялись в соотношении $1: 1$. Результаты моделирования для сплошного карбида UC и расчеты для компонентов приведены на рис. 6. Там же для иллюстрации приведены экспериментальные данные компонентов [39-41]. Для урана использовались данные экспериментов для материалов со значениями плотности, отличающимися от плотности монолитных образцов меныше $1 \%$.

Аналогичные расчеты выполнены для карбида молибдена $\mathrm{Mo}_{2} \mathrm{C}$, имеющего высокое значение температуры плавления. Объемные доли для этого карбида определялись в соотношении $2: 1$. Результаты моделирования для сплошного карбида $\mathrm{Mo}_{2} \mathrm{C}$ и расчеты для компонентов, а также экспериментальные данные компонентов приведены на рис. 7.

Полученные результаты показывают возможность описания динамических параметров (давление, сжатие) сплошных и пористых карбидов с различными стехиометрическими соотношениями при ударно-волновом нагружении. Для моделирования пористых образцов карбидов соотношение объемных долей конденсированных компонентов необходимо уменьшить пропорционально значению пористости.

Представляет интерес сравнение значения температуры вдоль ударной адиабаты $\mathrm{B}_{4} \mathrm{C}$ различных значений пористости с одним из результатов [43], где приведе-

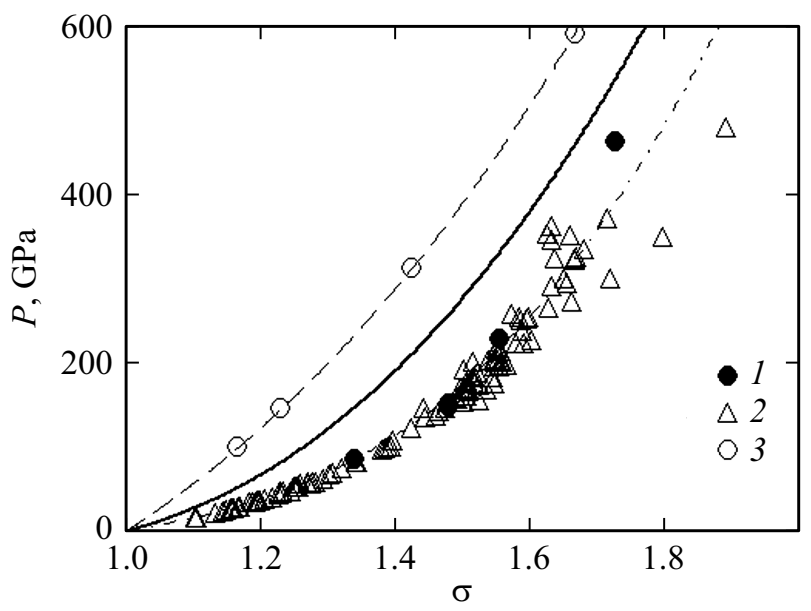

Рис. 6. Ударные адиабаты: штрихова линия для алмаза, штрихпунктирная линия для урана, сплошная линия для карбида урана UC. Экспериментальные данные: $1-m=1.006$ [41]; $2-m=1.005$ [39], $3-[40]$.

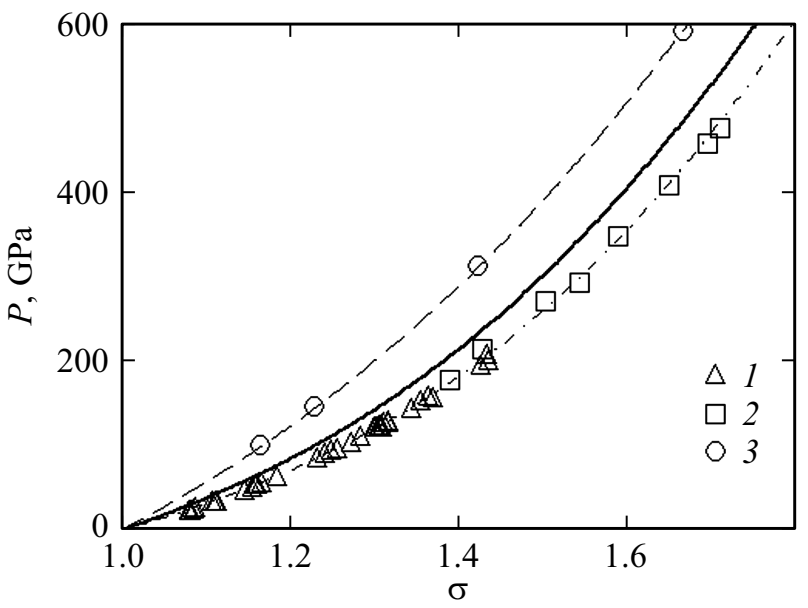

Рис. 7. Ударные адиабаты: штриховая линия для алмаза, штрихпунктирная линия для молибдена, сплошная линия для карбида молибдена $\mathrm{Mo}_{2} \mathrm{C}$. Экспериментальные данные: $1-[41] ; 2-[39], 3-[40]$. 


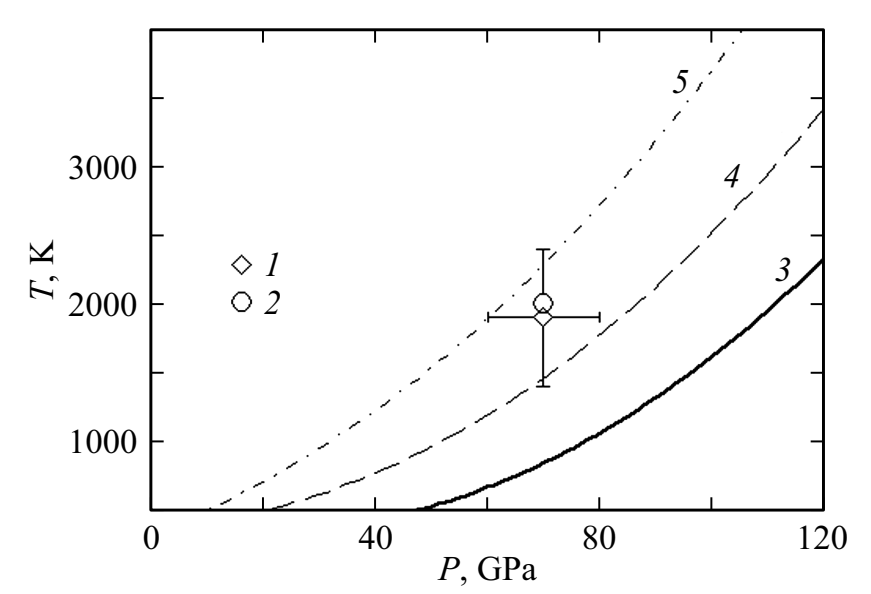

Рис. 8. Значение температуры в зависимости от давления вдоль ударной адиабаты для карбида бора. Данные: $1-[43]$, 2 - [44]. Расчет: сплошная линия 1 для $m=1$, штриховая линия 4 для $m=1.03$, штрихпунктирная линия 5 для $m=1.07$.

но значение температуры при давлении $70 \mathrm{GPa}$, pacсматриваемого как область фазового перехода, и данными молекулярно-динамического моделирования [44] (см. рис. 5 в [43]) для ударно-сжатого карбида бора. Модельный расчет и данные из $[43,44]$ показаны на рис. 8 . Дополнительно добавлены результаты моделирования для значений пористости $m=1.03,1.07$. Как видно из приведенных расчетов, для сплошного карбида $m=1$ значения температуры при $70 \mathrm{GPa}$ меньше полученных в [43,44], при этом они находятся в области между адиабатами, соответствующими значениям пористости $m=1.03,1.07$, что можно трактовать как область ударной адиабаты фазы высокого давления. Как было показано для углерода [45], для которого фазой высокого давления считается алмаз, экспериментальные значения выше области фазового перехода находятся близко к ударной адиабате алмаза той же начальной плотности, что и исследуемые образцы углерода. Это означает, что необходимо рассматривать ударную адиабату для фазы высокого давления с плотностью меньше монолитной. Таким образом, ударная адиабата карбида с пористостью $m=1.05$ с точностью 0.02 может рассматриваться как соответствующая фазе высокого давления, этим косвенно подтверждая возможность фазового перехода при этом значении давления.

В уравнении состояния (3) данной модели используется постоянное значение теплоемкости для компонентов. Данное предположение, как отмечено [46], можно считать оправданным при значениях сжатия меньше 2. Это приводит к некоторому завышению значения температуры исследуемых образцов, но тем не менее получено достоверное описание значений динамических параметров нитридов, таких, как давление и сжатие в диапазоне значений давления от 3 до $800 \mathrm{GPa}$. Следует отметить, что данная модель позволяет описать значения давления, сжатия и температуры нагружаемой среды, включая смеси, компоненты которых испытывают полиморфный фазовый переход $[47,48]$. Принимая во внимание возможный фазовый переход углерода, о котором упоминается в [26], где высказывалось предположение, что „на положение начального участка зависимостей волновая-массовая скорость может налагать и переход углерода в алмазную фазу, который, судя по всему, имеет место в этих условиях“, можно ожидать, что удастся достоверно описывать ударно-волновое нагружение в том числе и для карбидов, испытывающих фазовый переход при высокоэнергетическом воздействии.

\section{Заключение}

Таким образом, показано, что предложенная модель позволяет описывать динамическое нагружение карбидов различных значений пористости, рассматривая их как смесь компонентов, соответствующих химическому составу карбидов. Получено достоверное описание как для карбидов с равными долями 1:1 в стехиометрическом соотношении, так и для карбида бора, имеющего соответственно соотношение $1: 4$. По этой же методике можно моделировать термодинамические параметры карбидов с различными стехиометрическими соотношениями, для которых отсутствуют экспериментальные данные. Данный подход может быть полезен при численном моделировании поведения сложных материалов, в частности карбидов при высоких плотностях энергии.

\section{Конфликт интересов}

Автор заявляет, что у него нет конфликта интересов.

\section{Список литературы}

[1] F.A. Akopov, M.A. Adrianov, R.Kh. Amirov, T.I. Borodina, L.B. Borovkova, G.E. Val'yano, A.Yu. Dolgoborodov, V.V. Tkachenko, M.B. Shavelkina. Refract. Ind. Ceram., 57 (5), 496 (2017). DOI: 10.1007/s11148-017-0011-5

[2] А.А. Баканова, В.А. Бугаева, И.П. Дудоладов, Р.Ф. Трунин. Изв. АН СССР. Сер. Физика Земли, 6, 58 (1995).

[3] М.Н. Павловский. ФТТ, 12 (7), 2175 (1970).

[4] R.G. McQueen, S.P. Marsh, J.W. Taylor, J.N. Fritz, W.J. Carter. The Equation of State of Solids from Shock Wave Studies / In: High Velocity Impact Phenomena, ed. by R. Kinslow (Academic Press, NY., 1970)

[5] W.H. Gust, E.B. Royce. J. Appl. Phys., 42, 276 (1971). DOI: $10.1063 / 1.1686902$

[6] D. Grady. J. Phys. IV Proceedings, EDP Sci., 04 (C8), C8385-C8-391 (1994). DOI: 10.1051/jp4:1994859

[7] T.J. Vogler, W.D. Reinhart, L.C. Chhabildas. J. Appl. Phys., 95, 4173 (2004). DOI: 10.1063/1.1686902

[8] Y. Zhang, T. Mashimo, Y. Uemura, M. Uchino, M. Kodama, K. Shibata, K. Fukuoka, M. Kikuchi, T. Kobayashi, T. Sekine. J. Appl. Phys., 100, 113536 (2006). DOI: 10.1063/1.2399334

[9] D.E. Grady. J. Appl. Phys., 117, 165904 (2015). DOI: $10.1063 / 1.4918604$ 
[10] P. Dera, M.H. Manghnani, A. Hushur, Yi. Hu, S. Tkachev. J. Solid State Chem., 215, 85 (2014).

DOI: $10.1016 /$ j.jssc.2014.03.018

[11] S.A. Dyachkov, A.N. Parshikov, M.S. Egorova, S.Yu. Grigoryev, V.V. Zhakhovsky, S.A. Medin. J. Appl. Phys., 124, 085902 (2018). DOI: 10.1063/1.5043418

[12] D.E. Fratanduono, P.M. Celliers, D.G. Braun, P.A. Sterne, S. Hamel, A. Shamp, E. Zurek, K.J. Wu, A.E. Lazicki, M. Millot, G.W. Collins. Phys. Rev. B, 94, 184107 (2016). DOI: 10.1103/PhysRevB.94.184107

[13] А.М. Молодец, А.А. Голышев, Д.В. Шахрай, ЖЭТФ, 151 (3), 550 (2017). DOI: 10.7868/S0044451017030000 [A.M. Molodets, A.A. Golyshev, D.V. Shakhrai. J. Exp. Theor. Phys., 80, 467 (1995). DOI: 10.1134/S1063776117030049]

[14] K.K. Maevskii. AIP Conf. Proc., 2167, 020204 (2019). DOI: $10.1063 / 1.5132071$

[15] А.С. Савиных, И.А. Черепанов, С.В. Разоренов, А.И. Овсиенко, В.И.Румянцев, С.С. Орданьян. ЖТФ, 88 (12), 1813 (2018). DOI: 10.21883/JTF.2018.12.46782.3818 [A.S. Savinykh, I.A. Cherepanov, S.V. Razorenov, A.I. Ovsienko, V.I. Rumyantsev, S.S. Ordan'yan. Tech. Phys., 63, 1755 (2018). DOI: 10.1134/S1063784218120186]

[16] P.X. Баграмов, Н.P. Серебряная, В.М. Прохоров, В.Д. Бланк. ЖТФ, 88 (7) 1041 (2018). DOI: 10.21883/JTF.2018.07.46174.2545 [R.Kh. Bagramova, N.R. Serebryanaya, V.M. Prokhorov, V.D. Blank. Tech. Phys., 63 (7), 1010 (2018). DOI: 10.1134/S1063784218070046]

[17] А.С. Савиных, Г.В. Гаркушин, С.В. Разоренов, В.И. Румянцев. ЖТФ, 85 (6), 77 (2015). [A.S. Savinykh, G.V. Garkushin, S.V. Razorenov, V.I. Rumyantsev. Tech. Phys., 60, 863 (2015). DOI: $10.1134 / \mathrm{S} 1063784215060249]$

[18] А.И. Савватимский, С.В. Онуфриев. ТВТ, 58 (6), 865 (2020). DOI: 10.31857/S0040364420060186 [A.I. Savvatimskii, S.V. Onufriev. High Temp., 58, 800 (2020). DOI: /10.1134/S0018151X20060188]

[19] B.D. Sahoo, K.D. Joshi, T.C. Kaushik. Comput. Condens. Matter., 21, e00431 (2019).

DOI: 10.1016/j.cocom.2019.e00431

[20] J.S. Olsen, L. Gerward, U. Benedict, J.-P. Itié, K. Richter. J. Less Common Metal., 121, 445 (1986). DOI: $10.1016 / 0022-5088(86) 90561-8$

[21] B.D. Sahoo, K.D. Joshi, Satish C. Gupta, J. Nucl. Mater., 437, 81 (2013). DOI: 10.1016/j.jnucmat.2013.01.314

[22] B.D. Sahoo, D. Mukherjee, K.D. Joshi, T.C. Kaushik. J. Appl. Phys., 120, 085902 (2016). DOI: 10.1063/1.4961497

[23] J.-P. Dancausse, S. Heathman, U. Benedict, L. Gerward, J. Staun Olsen, F. Hulliger J. Alloy. Compd., 191, 309 (1993). DOI: 10.1016/0925-8388(93)90084-Z

[24] V.N. Senchenko, R.S. Belikov. J. Phys.: Conf. Ser., 1147, 012011. (2019). DOI: 10.1088/1742-6596/1147/1/012011

[25] А.С. Савиных, И.А. Черепанов, С.В. Разоренов, K. Mandel, L. Kruger. ЖТФ, 89(3), 392 (2019). DOI: $10.21883 /$ JTF.2019.03.47174.263-18 [A.S. Savinykh, I.A. Cherepanov, S.V. Razorenov, K. Mandel, L. Krüger. Tech. Phys., 64, 356 (2019). DOI: 10.1134/S1063784219030216]

[26] Р.Ф. Трунин. Исследования экстремальных состояний конденсированных веществ методом ударных волн. Уравнения Гюгонио (РФЯЦ-ВНИИЭФ, Саров, 2006), c. 137.
[27] А.Я. Пак, Т.Ю. Якич, Г.Я. Мамонтов, М.А. Рудмин, Ю.3. Васильева. ЖТФ, $90(5) \quad 805 \quad$ (2020). DOI: 10.21883/JTF.2020.05.49182.8-19 [A.Ya. Pak, T.Yu. Yakich, G.Ya. Mamontov, M.A. Rudmin, Yu.Z. Vasil'eva. Tech. Phys., 65, 771 (2020). DOI: $10.1134 / \mathrm{S} 1063784220050205]$

[28] S.A. Rasakia, B. Zhanga, K. Anbalgamb, T. Thomas, M. Yang. Prog. Solid State Chem., 50, 1 (2018) DOI: 10.1016/j.progsolidstchem.2018.05.001

[29] D. Cho, J.H. Park, Y. Jeong, Y.L. Loo. Ceram. Int., 41, 10974 (2015) DOI: 10.1016/j.ceramint.2015.05.041

[30] Q. Dong, M. Huang, C. Guo, G. Yu, M. Wu. Int. J. Hydrogen Energy, 42, 3206 (2017) DOI: 10.1016/j.ijhydene.2016.09.217

[31] А.Н. Ищенко, С.А. Афанасьева, Н.Н. Белов, В.В. Буркин, С.В. Галсанов, В.3. Касимов, В.А. Кудрявцев, Я.Д. Липатникова, Л.С. Марцунова, К.С. Рогаев, А.Ю. Саммель, А.Б. Скосырский, Н.Т. Югов. ЖТФ, 90 (3), 434 (2020) DOI: 10.21883/JTF.2020.03.48928. 226-19 [A.N. Ishchenko, S.A. Afanas'eva, N.N. Belov, V.V. Burkin, S.V. Galsanov, V.Z. Kasimov, V.A. Kudryavtsev, Ya.D. Lipatnikova, L.S. Martsunova, K.S. Rogaev, A.Yu. Sammel', A.B. Skosyrskii, N.T. Yugov. Tech. Phys., 65, 414 (2020). DOI: 10.1134/S106378422003010X]

[32] А.C. Савиных, K. Mandel, C.В. Разоренов, L. Kruger. ЖТФ, 88 (3), 368 (2018) DOI: 10.21883/JTF.2018.03.45592.2267 [A.S. Savinykh, K. Mandel, S.V. Razorenov, L. Krüger. Tech. Phys., 63, 357 (2018). DOI: 10.1134/S1063784218030210]

[33] K.K. Maevskii. J. Phys. Conf. Series., 894, 012057 (2017). DOI: 10.1088/1742-6596/894/1/012057

[34] К.К. Маевский, С.А. Кинеловский. ТВТ, 56 (6), 876 (2018). DOI: $10.31857 / \mathrm{S} 004036440003564-4$ [K.K. Maevskii, S.A. Kinelovskii. High Temperature, 56(6) 853 (2018). DOI: $10.1134 / \mathrm{S} 0018151 \mathrm{X} 18060172]$

[35] K.K. Maevskii, S A Kinelovskii. J. Phys. Conf. Series., 946, 012113 (2018). DOI: 10.1088/1742-6596/946/1/012113

[36] K.K. Maevskii. Math. Montis., 41, 123 (2018).

[37] К.К. Маевский. ЖТФ, 91 (5), 815 (2021). DOI: 10.21883/JTF.2021.05.50694.293-20

[K.K. Maevskii. Tech. Phys., 66, 791 (2021). DOI: $10.1134 / \mathrm{S} 1063784221050145]$

[38] Я.Б. Зельдович, Ю.П. Райзер. Физика ударных волн и высокотемпературных гидродинамических явлений (Физматлит, М., 2008), с. 519.

[39] P.R. Levashov, K.V. Khishchenko, I.V. Lomonosov, V.E. Fortov. AIP Conf. Proc., 706, 87 (2004). http://www.ihed.ras.ru/rusbank/

[40] М.Н. Павловский, ФТТ, 13 (3), 893 (1970).

[41] S.P. Marsh (editor). LASL Shock Hugoniot Data (Univ. California Press, Berkeley, 1980)

[42] Р.Ф. Трунин, Л.Ф. Гударенко, М.В. Жерноклетов, Г.В. Симаков. Экспериментальные данные по ударноволновому сжсатию и адиабатическому расширению конденсированных веществ (РФЯЦ-ВНИИЭФ, Саров, 2006)

[43] А.М. Молодец, А.А. Голышев, Г.В. Шилов. Письма в ЖЭТФ, 111 (12), 838 (2020). DOI: $10.31857 / \mathrm{S} 1234567820120101$

[A.M. Molodets, A.A. Golyshev, G.V. Shilov. JETP Lett., 111 (12), 720 (2020). DOI: 10.1134/S0021364020120103]

[44] M. DeVries, G. Subhash, A. Awasthi. Phys. Rev. B, 101, 144107 (2020). DOI: 10.110 
[45] И.В. Ломоносов, В.Е. Фортов, А.А. Фролова, К.В. Хищенко, А.А. Чарахчьян, Л.В. Шуршалов. ЖТФ, 73 (6), 66 (2003). [I.V. Lomonosov, V.E. Fortov, A.A. Frolova, K.V. Khishchenko, A. A. Charakhchyan, L.V. Shurshalov. Tech. Phys., 48, 727 (2003). DOI: 10.1134/1.1583826]

[46] А.В. Острик. Конструкции из композиционных материалов, 2, 48 (2018).

[47] K.K. Maevskii, S.A. Kinelovskii. AIP Conf. Proc., 1783, 020143 (2016). DOI: 10.1063/1.4966436

[48] K.K. Maevskii. Math. Montis., 50, 140 (2021).

DOI: $10.20948 /$ mathmontis-2021-50-12 